\title{
Dementia is a predictor for mortality outcome from coronavirus disease 2019 (COVID-19) infection
}

\author{
Timotius Ivan Hariyanto ${ }^{1} \cdot$ Cynthia Putri ${ }^{1} \cdot$ Rocksy Fransisca V. Situmeang ${ }^{2} \cdot$ Andree Kurniawan $^{3}$ (i)
}

Received: 6 August 2020 / Accepted: 14 October 2020 / Published online: 26 October 2020

c) Springer-Verlag GmbH Germany, part of Springer Nature 2020

\section{Dear Editor,}

Coronavirus disease 2019 (COVID-19), a pandemic disease, has caused a significant impact on the economic, health, and social aspect. The disease has caused over 500,000 deaths around the world. Therefore, identification of the contributing factors to the development of mortality is very important to enable stratification of risk factors, optimize the reallocation of hospital resources, and guide public health recommendations and interventions. Before this pandemic, individuals with dementia are one of the most vulnerable persons whose day-to-day survival depends on other people. In the previous meta-analysis, it has been shown that patients with dementia have a two-fold risk of pneumonia-associated death [1]. However, until recently, no study provides clear evidence regarding the link between dementia and COVID19. The aim of this article is to explore the potential association between dementia and the mortality of COVID-19 infection.

A search of the literature was conducted on PubMed and PubMed Central (PMC) using the keywords "dementia" OR "clinical characteristics" OR "comorbidities" OR "risk factors" AND "coronavirus disease 2019" OR "COVID-19", between 2019 and present time (July 14th, 2020) with language restricted to English only. The title, abstract, and full text of all articles identified that matched the search criteria were assessed, and those reporting the rate of dementia in

Andree Kurniawan

andree.kurniawan@uph.edu

1 Faculty of Medicine, Pelita Harapan University, Boulevard Jendral Sudirman street, Karawaci, Tangerang 15811, Indonesia

2 Memory Clinic, Department of Neurology, Siloam Hospitals Lippo Village, Boulevard Jendral Sudirman street, Karawaci, Tangerang 15811, Indonesia

3 Department of Internal Medicine, Faculty of Medicine, Pelita Harapan University, Boulevard Jendral Sudirman street, Karawaci, Tangerang 15811, Indonesia
COVID-19 patients with a clinically validated definition of "mortality" were included in this meta-analysis. The references of all identified studies were also analyzed (forward and backward citation tracking) to identify other potentially eligible articles.

A meta-analysis was performed using Review Manager 5.4 (Cochrane Collaboration) software. Dichotomous variables were calculated using the Mantel-Haenszel formula with random-effects models regardless of its heterogeneity. The effect estimate was reported as risk ratio (RR) along with its $95 \%$ confidence intervals (CIs) for dichotomous variables, respectively. $P$ value was two-tailed, and the statistical significance set at $\leq 0.05$.

A total of 32,509 records were obtained through systematic electronic searches and other ways. After screening titles, abstracts, and full texts, 5 studies [2-6] with a total of 881 COVID-19 patients were included in the meta-analysis. The essential characteristics of included studies are summarized in Table 1, whilst the individual and pooled RRs for dementia predicting severe COVID-19 is shown in Fig. 1. Our pooled analysis showed that dementia significantly associated with an increased risk of mortality from COVID-19 [RR 2.60 (95\% CI 1.86-3.65), $p<0.00001, I^{2}=44 \%$, random-effect modelling].

Based on our meta-analysis of available data, dementia seems to be associated with an enhanced risk of mortality from COVID-19 infection. Several reasons can be proposed to explain this result. First, most of the patients with dementia were old in age and have other comorbid medical conditions that could increase the severity and mortality of infections. Older patients with COVID-19 infection may present with atypical symptoms of infections. They may be afebrile with non-respiratory symptoms, such as delirium or isolated functional decline without any obvious physical symptoms [7]. These atypical presentations of COVID-19 may impede the early recognition of the disease, increase COVID-19 spread, and mortality. Moreover, metaanalysis has also shown that older age ( $\geq 65$ years old $)$ and 
Table 1 Characteristics of included studies

\begin{tabular}{|c|c|c|c|c|c|c|}
\hline \multirow[t]{2}{*}{ Study } & \multirow[t]{2}{*}{ Sample size } & \multirow[t]{2}{*}{ Design } & \multicolumn{2}{|c|}{ Survivor patients } & \multicolumn{2}{|c|}{ Non-survivor patients } \\
\hline & & & $n(\%)$ & Age (years) & $n(\%)$ & Age (years) \\
\hline Bianchetti et al. [2] & 627 & Retrospective cohort & $433(69.1 \%)$ & N/A & $194(30.9 \%)$ & N/A \\
\hline Covino et al. [3] & 69 & Retrospective cohort & $46(66.7 \%)$ & $84.6 \pm 5.9$ & $23(33.3 \%)$ & $84.6 \pm 2.2$ \\
\hline Hwang et al. [4] & 103 & Retrospective cohort & $77(75 \%)$ & $64.6 \pm 15.8$ & $26(25 \%)$ & $76.5 \pm 9.2$ \\
\hline Wan et al. [5] & 30 & Retrospective cohort & $25(83.3 \%)$ & $62.7 \pm 13.6$ & $5(16.7 \%)$ & $66.8 \pm 5.1$ \\
\hline Yan et al. [6] & 52 & Retrospective cohort & $20(38.4 \%)$ & $51.9 \pm 12.9$ & $32(61.6 \%)$ & $64.6 \pm 11.2$ \\
\hline
\end{tabular}

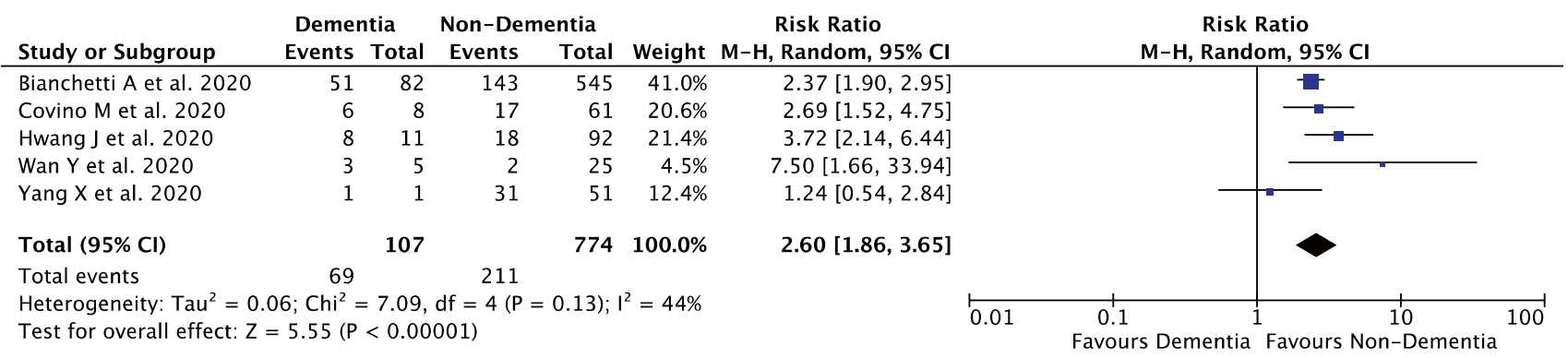

Fig. 1 Forest plot that demonstrates the association of dementia with mortality from COVID-19 disease

the presence of comorbid diseases were associated with a higher risk of mortality from COVID-19 infections [8]. Second, there is an ApoE e4 genotype which was associated with dementia. This genotype has a role to modulate the pro/anti-inflammatory phenotypes of macrophage. Besides expressing ACE2, the receptor for SARS-CoV-2, many cell types in the lung, such as macrophages, type I and type II alveolar epithelial cells, also express the ApoE e4 genotype [9]. Therefore, it is possible that having one or two copies of ApoE4 can make the robust innate immune response and cytokine storm which contribute to the development of COVID-19 complications, such as acute respiratory distress syndrome (ARDS), and lead to mortality. A study by Kuo et al. [10] have also demonstrated that patients with ApoE e4e4 were more likely to have severe COVID-19 infections that increase the risk of mortality.

Hence, patients with dementia should be given extra care and monitoring to minimize exposure to the virus. Physicians can use the telemedicine-based practice to provide care and evaluations for dementia patients to minimize their visit to the hospitals. Physicians and caregivers should also be engaged in close monitoring of dementia patients with suspected COVID-19, for early diagnosis and treatment to avoid mortality from infections. The hospitals can provide a Special Care Geriatric COVID-19 units for dementia and elderly patients who contract with COVID-19, which is filled with a team of mental health professionals (doctors, nurses, and psychological counselors), to give better care, more strict monitoring, and enable the healthcare providers in that units to focus only on the management of dementia patients so that the morbidity and mortality from COVID-19 can be reduced. Finally, dementia should be regarded as an important factor in future risk stratification models for COVID-19.

The limitation of this study is that the presence of confounding factors, such as patients' nutritional status and daily medication, that can affect the relationship between dementia and mortality from COVID-19 should still be considered. Moreover, this study only includes a small number of patients due to most studies still did not consider dementia as an important comorbidity in COVID-19 patients. However, with this study, we hope that dementia can further be considered as important factor and comorbidity in COVID19 patients.

Author contributions TIH: Conceptualization; Data curation; Methodology; Investigation; Validation; Visualization; Writing-original draft; Writing-review and editing. CP: Conceptualization; Data curation; Methodology; Investigation; Validation; Visualization; Writingoriginal draft; Writing-review \& editing. RFVS: Conceptualization; Validation; Resources; Writing-original draft; Writing-review and editing; Supervision. AK: Conceptualization; Validation; Resources; Writing-original draft; Writing-review and editing; Supervision.

Funding None.

Data availability Not applicable.

\section{Compliance with ethical standards}

Conflict of interest The authors declare that they have no conflict of interest. 


\section{References}

1. Manabe T, Fujikura Y, Mizukami K, Akatsu H, Kudo K (2019) Pneumonia-associated death in patients with dementia: a systematic review and meta-analysis. PLoS ONE 14(3):e0213825. https ://doi.org/10.1371/journal.pone.0213825

2. Bianchetti A, Rozzini R, Guerini F, Boffelli S, Ranieri P, Minelli $\mathrm{G}$ et al (2020) Clinical presentation of COVID19 in dementia patients. J Nutr Health Aging 24(6):560-562. https://doi. org/10.1007/s12603-020-1389-1

3. Covino M, Matteis GD, Santoro M, Sabia L, Simeoni B, Candelli $\mathrm{M}$ et al (2020) Clinical characteristics and prognostic factors in COVID-19 patients aged $\geq 80$ years. GeriatrGerontolInt 24(6):560-562

4. Hwang JM, Kim JH, Park JS, Chang MC, Park D (2020) Neurological diseases as mortality predictive factors for patients with COVID-19: a retrospective cohort study. NeurolSci. https://doi. org/10.1007/s10072-020-04541-z

5. Wan Y, Wu J, Ni L, Luo Q, YuanFan CF et al (2020) Prognosis analysis of patients with mental disorders with COVID-19: a single-center retrospective study. Aging 12(12):11238-11244. https://doi.org/10.18632/aging.103371
6. Yang X, Yu Y, Xu J, Shu H, Xia J, Liu H et al (2020) Clinical course and outcomes of critically ill patients with SARS-CoV-2 pneumonia in Wuhan, China: a single-centered, retrospective, observational study. Lancet Respir Med 8(5):475-481. https:// doi.org/10.1016/S2213-2600(20)30079-5

7. D'Adamo H, Yoshikawa T, Ouslander J (2020) Coronavirus disease 2019 in geriatrics and long-term care: the ABCDs of COVID -19. J Am GeriatrSoc 68(5):912-917. https://doi.org/10.1111/ jgs. 16445

8. Parohan M, Yaghoubi S, Seraji A, Javanbakht MH, Sarraf P, Djalali M, Risk factors for mortality in patients with Coronavirus disease, (2019) (COVID-19) infection: a systematic review and meta-analysis of observational studies. Aging Male 2020:1-9. https://doi.org/10.1080/13685538.2020.1774748

9. Kuo C, Pilling LC, Atkins JL, Kuchel GA, Melzer D (2020) ApoE e2 and aging-related outcomes in 379,000 UK Biobank participants. Aging (Albany NY) 12:12222-12233. https://doi. org/10.18632/aging.103405

10. Kuo CL, Pilling LC, Atkins JL, Masoli JAH, Delgado J, Kuchel GA et al (2020) APOE e4 genotype predicts severe COVID-19 in the UK Biobank community cohort. J Gerontol A BiolSci Med Sci. https://doi.org/10.1101/2020.05.07.20094409 